JACQUELINE ALVES LEITE

\title{
AVALIAÇÃO DO PAPEL MODULADOR DA OUBAÍNA NO EIXO HIPOTALÂMICO-PITUITÁRIO-ADRENAL EM RATOS SUBMETIDOS AO ESTRESSE CRÔNICO IMPREVISÍVEL
}

Tese apresentada ao programa de Pós-Graduação em Farmacologia do Instituto de Ciências Biomédicas da Universidade de São Paulo para a obtenção do Título de Doutor em Ciências

Área de concentração: Farmacologia

Orientador: Prof. Dr. Cristoforo Scavone

Versão Original

São Paulo

2018 


\section{Resumo}

Leite JA. Avaliação do papel modulador da ouabaína no eixo hipotalâmico-pituitárioadrenal em ratos submetidos ao estresse crônico imprevisível. [Tese (Doutorado em Farmacologia)]. São Paulo: Instituto de Ciências Biomédicas, Universidade de São Paulo, São Paulo; 2018.

A ouabaína (OUA), um inibidor da $\mathrm{Na}^{+}, \mathrm{K}^{+}-\mathrm{ATPase}$, foi identificada como uma substância endógena presente no plasma humano, e parece estar envolvida na resposta ao estresse agudo, em animais e seres humanos. O estresse crônico é um importante fator agravante de doenças psiquiátricas, incluindo depressão e ansiedade. Além disso, problemas cognitivos são cada vez mais reconhecidos como importantes componentes da ansiedade e depressão. Diante disto, o presente trabalho buscou investigar os efeitos da OUA $(1,8 \mu \mathrm{g} / \mathrm{kg})$ na hiperatividade do eixo HPA, na neuroinflamação, na expressão de receptores e proteínas envolvidos na plasticidade sináptica, nos efeitos comportamentais (como déficit de memória de longa duração, depressão e ansiedade) e atividade da $\mathrm{Na}^{+}, \mathrm{K}^{+}-$ATPase induzidos pelo protocolo de estresse crônico imprevisível (CUS) realizado ao longo de 14 dias em ratos. Nossos resultados demonstraram que o tratamento intermitente com OUA é capaz de reverter a hiperatividade do eixo HPA induzido pelo CUS, por meio da redução de glicocorticoide, redução na expressão de $\mathrm{CRH}-\mathrm{CRHR} 1$, bem como diminuir a neuroinflamação, e aumentar os níveis de BDNF e fazer o que na expressão dos receptores CRHR2. Essas alterações bioquímicas contribuíram para uma reversão nos prejuízos na memória de longo prazo induzida pelo CUS. Ademais os animais tratados apenas com OUA, bem como os submetidos ao CUS e tratados com OUA obtiveram uma melhora na memória emocional, averiguada no teste comportamental de condicionamento da memória ao medo. Os resultados encontrados sugerem que o protocolo de CUS por 14 dias promove uma adaptação neuronal facilitando a redesignação da memória ao medo, aqui configurado pelo choque, e o tratamento com a OUA abrevia esse processo. Em conclusão os nossos resultados sugerem que o tratamento intermitente com OUA suscita uma adaptação no eixo HPA, por meio de alterações na expressão dos receptores para $\mathrm{CRH}$ no hipocampo e hipotálamo, resultando em uma adaptação na memória emocional relacionada ao medo.

Palavras-Chaves: Estresse Crônico Imprevisível, Ouabaína, memória, eixo hipotálamo-pituitária-adrenal. 


\begin{abstract}
Leite JA. Evaluation of the role of ouabain in the hypothalamic-pituitary-adrenal axis in rats submitted to unpredictable chronic stress. [PhD Thesis (Pharmacology)]. São Paulo: Instituto de Ciências Biomédicas, Universidade de São Paulo, São Paulo; 2018.

Ouabain (OUA), an inhibitor of $\mathrm{Na}^{+}, \mathrm{K}^{+}-$ATPase, has been identified as an endogenous substance present in human plasma, and appears to be involved in the response to acute stress in animals and humans. Chronic stress is an important aggravating factor of psychiatric illness, including depression and anxiety. In addition, cognitive problems are increasingly recognized as important components of anxiety and depression. The present work aimed to investigate the effects of OUA $(1.8 \mu \mathrm{g} / \mathrm{kg})$ on HPA axis hyperactivity, neuroinflammation, expression of receptors and proteins involved in synaptic plasticity, behavioral effects (such as long-term memory deficit duration, depression and anxiety) and $\mathrm{Na}^{+}, \mathrm{K}^{+}$-ATPase activity induced by the unpredictable chronic stress protocol (CUS) performed over 14 days in rats. Our results demonstrated that intermittent treatment with OUA was able of reversing CUS-induced HPA axis hyperactivity, by reducing glucocorticoid levels, CRH-CRHR1 expression, as well as reducing CUS-induced low-grade neuroinflammation, and increase BDNF levels and expression of CRHR2 receptors. These biochemical changes contributed to a reversal in CUS-induced long-term memory impairment. In addition, animals treated only with OUA, as well as those submitted to CUS, and also treated with OUA obtained an improvement in emotional memory, which was explored in the fear conditioning test. These results suggest that the CUS protocol of 14 days promotes a neural adaptation facilitating a reassignment of the memory to the fear, here configured by the shock, and the treatment with the OUA shortens that process. In conclusion, our results suggest that intermittent treatment with OUA induces an adaptation on the HPA axis, through alterations in the expression of receptors for $\mathrm{CRH}$ in the hippocampus and hypothalamus, resulting in an adjustment in fear-related emotional memory
\end{abstract}

Keywords: Unpredictable chronic stress, Ouabain, memory, HPA axis 


\section{INTRODUÇÃO}

\subsection{Estresse}

A resposta ao estresse é um mecanismo de adaptação que permite ao organismo manter a homeostase durante desafios ambientais, sendo coordenada pela ativação do eixo hipotálamo-pituitária-adrenal (HPA). As mudanças em curto prazo que ocorrem no eixo HPA, preparam o organismo para enfrentar diferentes estímulos ambientais (CHARMANDARI; TSIGOS; CHROUSOS, 2005; GILLESPIE; NEMEROFF, 2005).

O eixo HPA é composto por um conjunto complexo de interações entre hipotálamo, glândula pituitária e córtex da adrenal, que medeia a resposta ao estresse através das ações de glicocorticóides. Em resposta ao estresse, o núcleo paraventricular (PVN) libera o hormônio liberador de corticotropina (CRH) e arginina vasopressina (AVP), que entram na circulação portal hipofisária e estimulam a síntese e secreção de hormônio adrenocorticotrófico (ACTH) para a circulação periférica. Quando o CRH e AVP ligam-se em seus respectivos receptores hormônio liberador de corticortrofina $(\mathrm{CRH}) 1$ e AVP-R1b, localizados em células corticotróficas da hipófise, induzem a produção de pró-opiomelanocortina (POMC), molécula precursora do ACTH e lipotropinas (LPH) (VALE et al., 1981).

O ACTH estimula a produção e secreção de esteróides nas glândulas adrenais, sendo o cortisol produzido em humanos e a corticosterona em roedores. Este processo é mediado pela interação do ACTH com seu receptor de melanocortina tipo 2 (MC2) no córtex da adrenal (TSIGOS; CHROUSOS, 2002). A regulação do eixo HPA é mantida por meio da atuação direta do cortisol ou corticosterona no PVN, inibindo por retroalimentação negativa a síntese de CRH e POMC (Figura 1) (CHARMANDARI; TSIGOS; CHROUSOS, 2005). Além disso, o cortisol age em regiões extrahipotalâmicas, tais como hipocampo e amígdala, mantendo a homeostase corporal (ZIEGLER, 2002). 
Figura 1 - Esquema de ativação e regulação do eixo HPA.

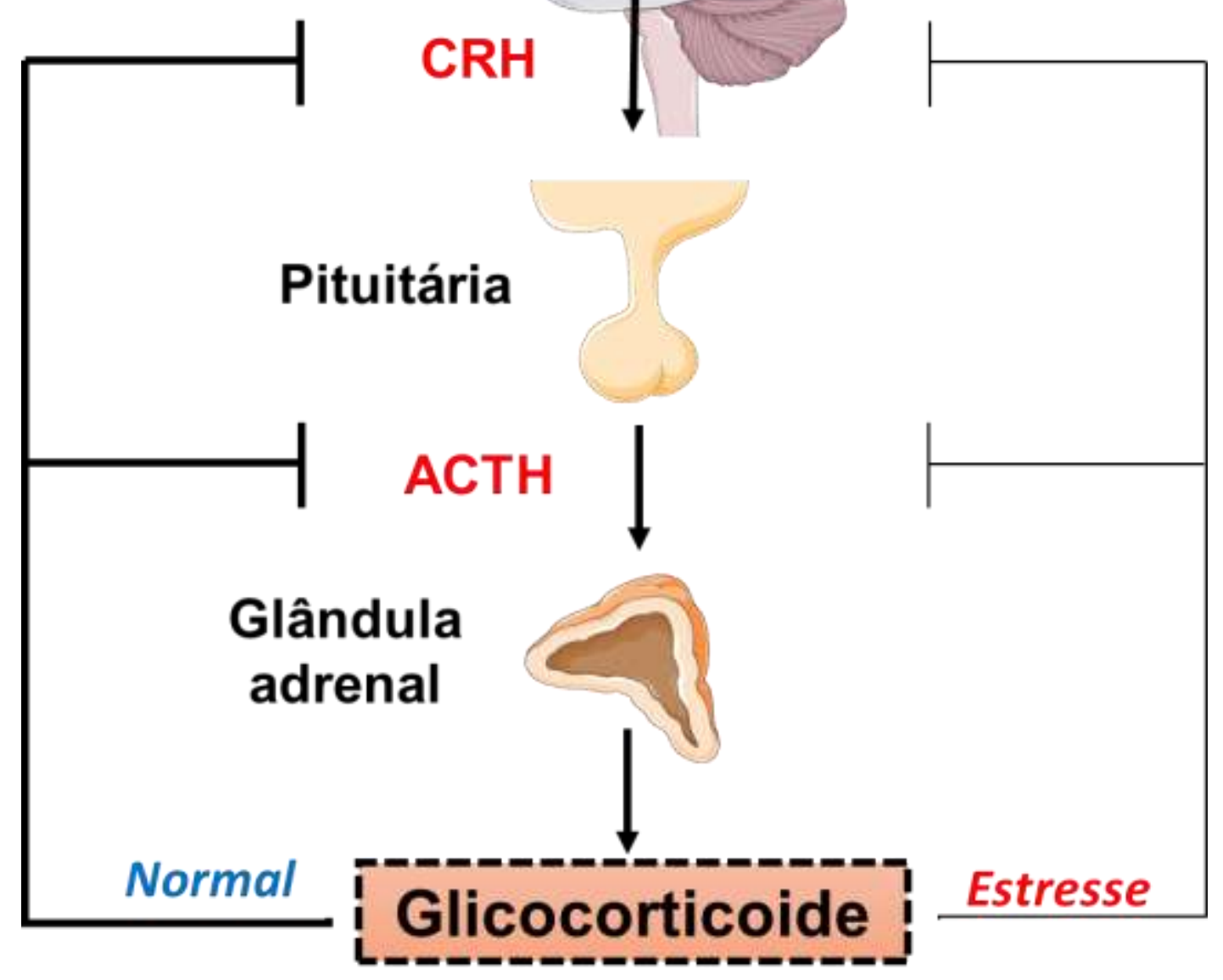

O CRH produzido no PVN do hipotálamo atuam estimulando a síntese e secreção de ACTH na pituitária anterior. O ACTH promove a produção e liberação de glicocorticoides (GCs) a partir do córtex adrenal. Em condições normais, a atividade do eixo HPA é controlada através de uma retroalimentação negativa dos GCs na pituitária, PVN e sistema límbico através da ativação dos receptores GR e $\mathrm{MR}$, inibindo a produção e liberação de $\mathrm{CRH}$. Durante o estresse crônico, esse controle exercido pela retroalimentação negativa está diminuído reforçando a liberação de glicocorticoide. 


\subsection{Glicocorticóides}

A secreção de glicocorticóides pelas adrenais é pulsátil, seguindo o ritmo circadiano que coordena e sincroniza acontecimentos diários e relacionados ao sono (LIGHTMAN et al., 2008). No entanto, o controle da liberação desse hormônio pode ser facilmente acometido pela perda da homeostasia fisiológica, pelo próprio processo de envelhecimento ou pelo estresse (YOUNG; ABELSON; LIGHTMAN, 2004).

Mediante uma situação de estresse, por exemplo, o glicocorticóide é capaz de afetar a liberação de glicose na circulação sanguínea, suprimir funções do sistema imunológico e afetar o processamento de informação nas redes neuronais límbicas envolvidas na emoção, cognição, e formação da memória (DE KLOET et al., 2009). Devido a estes efeitos deletérios da exposição prolongada de glicocorticóide, a atividade do eixo HPA é modulada por uma retroalimentação negativa induzida por glicocorticóides e ACTH que atuam no hipocampo, PVN e glândula pituitária (PAPADIMITRIOU; PRIFTIS, 2009).

Uma vez secretado na corrente sanguínea o cortisol encontra-se normalmente ligado à globulina ligadora de cortisol (CBG), e somente na sua forma livre atravessa facilmente a barreira hematoencefálica e membranas celulares. Os glicocorticóides se ligam a dois tipos de receptores nucleares que apresentam atividade de fatores de transcrição: o receptor de mineralocorticóide ou tipo I (MR) e o receptor de glicocorticóide ou tipo II (GR). O GR encontra-se amplamente expresso no SNC, enquanto que, o MR é expresso principalmente em áreas límbicas, tais como no hipocampo e amígdala (TER HEEGDE; DE RIJK; VINKERS, 2015). Os MRs apresentam maior afinidade para glicocorticóides que os GRs (revisado em Heeged, 2015), logo, os MRs estão envolvidos na manutenção basal do eixo HPA (ritmos circadianos), enquanto que os efeitos mediados pelos GRs são acionados principalmente em decorrência de situações de estresse, onde os níveis de glicocorticóide circulante encontram-se aumentados (TER HEEGDE; DE RIJK; VINKERS, 2015).

$\mathrm{Na}$ ausência de seus ligantes, os receptores para glicocorticóides encontramse no citoplasma na forma de um complexo multiprotéico, associados às proteínas de choque térmico (HSP90, HSP70 e HSP65). Estes complexos de proteínas são importantes para a estabilização dos receptores no citosol (DE KLOET et al., 1998; SCHESCHOWITSCH; LEITE; ASSREUY, 2017). Quando ativados, tanto os GRs 
quanto os MRs modulam a expressão gênica por mecanismos de transativação ou transrepressão. A transativação envolve o processo de homodimerização dos receptores, que, em seguida, ligam-se ao elemento responsivo aos glicocorticóides (GRE) no DNA para induzir ou inibir a transcrição, enquanto que a transrepressão ocorre por interação direta com outros fatores de transcrição como o NF-KB, CREB e proteína ativadora 1 (AP1), ambas as vias modulam a síntese de proteínas (SCHESCHOWITSCH; LEITE; ASSREUY, 2017).

O estresse agudo aumenta os níveis de glicocorticóides e receptores GR no cérebro, sendo que no córtex pré-frontal (PFC) esse aumento é capaz de suprimir a resposta do eixo HPA (BELDA et al., 2008; FUCHS; FLÜGGE, 2003). Entretanto, no estresse crônico níveis elevados circulantes de ACTH e glicocorticóide estão relacionados com alterações na expressão dos receptores GR e MR em diferentes regiões do sistema nervoso central (SNC), incluindo hipocampo, córtex pré-frontal e hipotálamo, e foi observado que após o período de recuperação ao estresse houve uma diminuição na expressão do RNAm de CRH no PVN, bem como para MR e GR no hipocampo e PVN (MARQUES; SILVERMAN; STERNBERG, 2009; SILVERMAN; STERNBERG, 2012).

Apesar de o glicocorticóide ter ações anti-inflamatórias bem estabelecidas no sistema periférico, estudos têm demonstrado que, durante o modelo de estresse crônico imprevisível (CUS), o aumento nos níveis de glicocorticóides pode potencializar a ativação do NF-kB induzida pela administração de lipopolissacarídeo (LPS), bem como expressão de citocinas pró-inflamatórias no córtex frontal e hipocampo, mas não no hipotálamo. Os efeitos pró-inflamatórios do CUS foram mediados via receptores $\mathrm{GR}$, uma vez que os efeitos de potencialização do estresse na ativação do NF-kB foram antagonizados na vigência de RU-486, um antagonista para receptores GR (MUNHOZ, 2006). Isso sugere que o estresse pode acelerar a neurodegeneração, provavelmente via aumento de corticosteróides de forma prolongada. Além disso, outros trabalhos mostraram que os glicocorticóides promovem um aumento no número de células inflamatórias, tais como granulócitos, monócitos/macrófagos e ativação da microglia no hipocampo, bem como a produção de citocinas como IL-1, IL-6 e TNF- $\alpha$ (DINKEL; MACPHERSON; SAPOLSKY, 2003; MUNHOZ et al., 2010).

No entanto, os efeitos dos glicocorticóides na neuroinflamação são controversos, visto que outros estudos demonstraram que os glicocorticóides inibem 
a ativação da microglia, reduzindo as reações inflamatórias no encéfalo (NADEAU; RIVEST, 2003; SUGAMA et al., 2009). Mais recentemente foi observado que o aumento de glicocorticóide durante o estresse agudo é capaz de promover um controle na ativação da microglia limitando sua capacidade em produzir mediadores envolvidos na inflamação (SUGAMA et al., 2013). Além disso, a administração de dexametasona parece suprimir a ativação do NF-KB induzida por LPS tanto perifericamente como no SNC (Munhoz et al., 2006). Estas ações paradoxais dos glicocorticóides sobre a ativação da microglia podem ser atribuídas aos diferentes modelos experimentais, às diferentes abordagens farmacológicas, ou às diferentes concentrações de hormônios esteroides (MACPHERSON; DINKEL; SAPOLSKY, 2005).

\subsection{Receptores de $\mathrm{CRH}$ e seus ligantes}

O CRH foi descoberto por Vale et al. (1981) como um neuropeptídeo endógeno formado por 41 resíduos de aminoácidos. Este neuropeptídeo encontra-se amplamente distribuído no SNC, predominantemente no PVN, PFC, núcleo central da amígdala, cerebelo e tronco cerebral (TSIGOS; CHROUSOS, 2002). A expressão de $\mathrm{CRH}$ é estimulada por neurotransmissores, como serotonina (Laflamme et al., 1999), norepinefrina (ITOI et al., 1994) e citocinas como fator de necrose tumoral (TNF)- $\alpha$, IL-1 e IL-6 (TURNBULL; RIVIER, 1999).

$\mathrm{O} \mathrm{CRH}$ liga-se aos receptores CRHR que existem em duas isoformas o Receptor 1 do hormônio liberador de corticotropina (CRHR1) e o Receptor 2 do hormônio liberador de corticotropina (CRHR2), ambos são receptores metabotrópicos pertencentes à família de receptores acoplados a proteína-G (GPCRs). A ativação dos receptores de CRF predominantemente leva à sinalização pela adenilato ciclase via Gs, promovendo o aumento dos níveis de AMPc, mas eles também podem sinalizar com outras proteínas $\mathrm{G}$, como $\mathrm{Gq} / 11$ e $\mathrm{G}_{0}$ dependendo especificamente do tecido onde se encontram (BLANK et al., 2003). Em células corticotróficas da pituitária, o receptor CRHR1 é o principal responsável pela regulação da síntese e secreção de ACTH, que por sua vez, estimula a libertação de glicocorticóides a partir do córtex adrenal (MAJZOUB, 2006). Por outro lado, a ativação do CRHR2 promove supressão do apetite e age como um antidepressivo. Assim, o CRHR1 participa da resposta 
normal ao estresse, enquanto que o $\mathrm{CRHR2}$ medeia uma resposta de ajuste ao estresse. Além disso, os dois receptores para $\mathrm{CRH}$ têm funções opostas na regulação do comportamento, onde o CRHR1 tem efeito ansiogênico e o CRHR2 efeito ansiolítico (BALE; VALE, 2004; MAJZOUB, 2006), conforme ilustrado na figura 2.

Além do $\mathrm{CRH}$, uma família de ligantes do receptor de $\mathrm{CRH}$ foi encontrada em mamíferos, denominados Urocortinas (Ucn) (Figura 2). As Ucns são neuropeptídeos com estruturas semelhante ao CRF e apresentam três isoformas diferentes: Ucn-1, Ucn-2 e Ucn-3, sendo expressas sistemicamente. A Ucn1 apresenta 45\% de homologia com o $\mathrm{CRH}$ sendo expressa no SNC em regiões como o núcleo de EdingerWestphal e hipotálamo (BITTENCOURT et al., 1999; MACPHERSON; DINKEL; SAPOLSKY, 2005; WONG et al., 1996), e em maior expressão nos sistemas periféricos, como trato gastrointestinal, testículos, coração (BOORSE; DENVER, 2006). 
Figura 2 - Esquema representativo dos receptores $\mathrm{CRH}$ e efeitos fisiológicos/ comportamentais decorrentes da sua ativação.
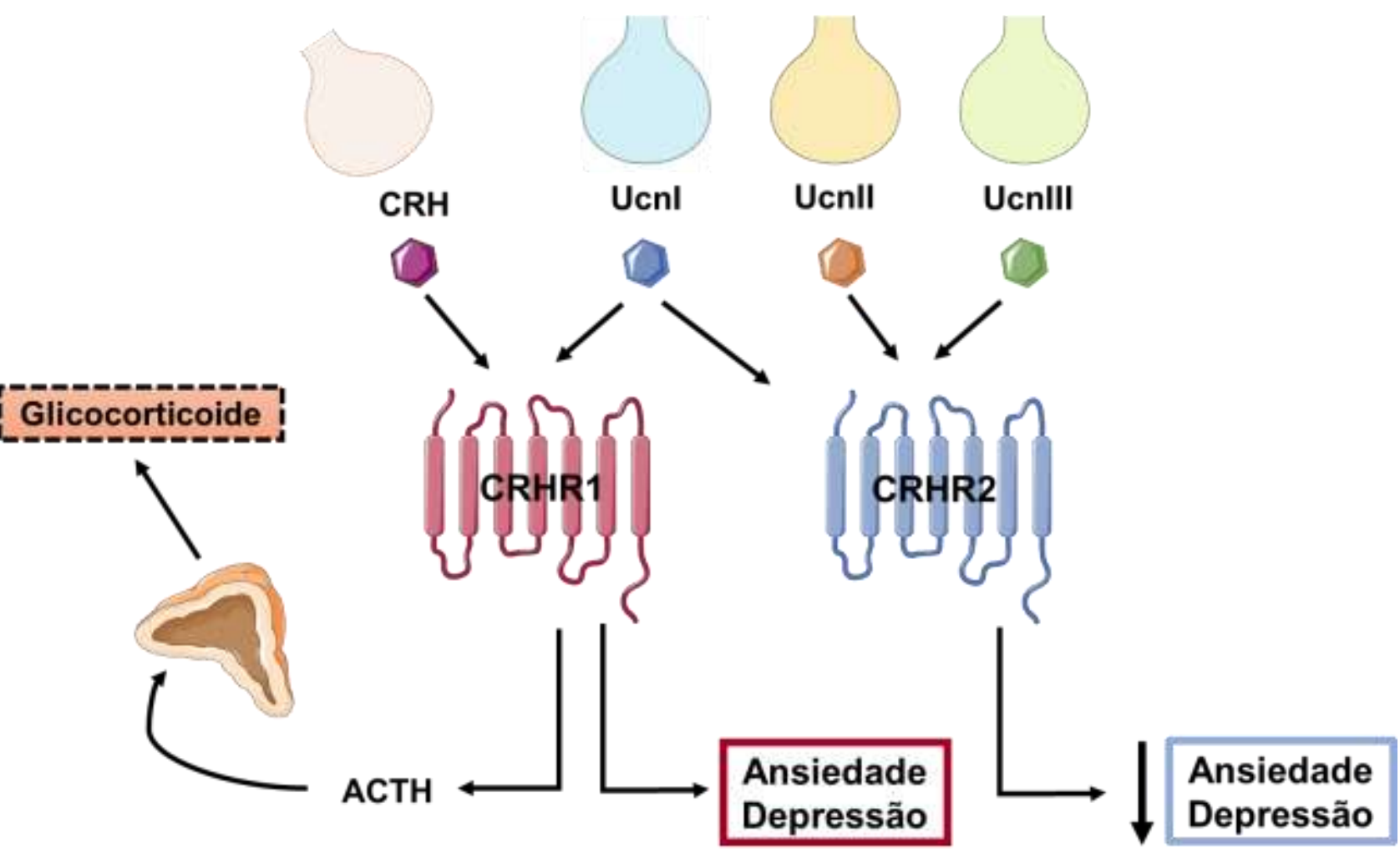

Ucnll UcnIII

O CRH apresenta baixa afinidade pelo CRHR2 em relação ao CRHR1. A ligação do hormônio liberador de corticotropina ao CRHR1 participa da regulação do eixo HPA, induzindo a produção de ACTH e glicocorticóide pela adrenal, bem como promove ativação do SNS (Sistema Nervoso Simpático) e favorece a depressão. No entanto, o CRHR2 promove supressão do apetite e induz efeitos antidepressivos. A Urucortina 1 (Ucnl) tem atividade semelhante nos receptores CRHR1 e CRHR2, enquanto que as Ucnll e Ucnll apresentam maior afinidade pelos receptor CRHR2. Adaptado de Bale e Vale (2004) (BALE; VALE, 2004). 


\subsection{Estresse e Inflamação}

Além das regulações neuro-endócrinas citadas anteriormente relacionadas ao eixo HPA, achados recentes mostram que o estresse infeccioso/inflamatório pode ativar o eixo HPA aumentando os níveis de ACTH e glicocorticóide plasmáticos, levando à supressão do sistema imunológico, prevenindo uma superativação da resposta inflamatória (BESEDOVSKY; DEL REY, 1996; CHESNOKOVA; MELMED, 2002). As citocinas são mediadores importantes da interação entre os sistemas neuroendócrino e imunológico.

A interleucina (IL)-1 $\beta$ apresenta um importante papel regulador do eixo HPA (GOSHEN; YIRMIYA, 2009) encontrando-se expressa em todas as regiões deste, interferindo na secreção de $\mathrm{CRH}$, ACTH e glicocorticóide a partir do hipotálamo, hipófise e córtex adrenal, respectivamente. Além disso, a IL- $1 \beta$, via ativação de seus receptores IL-1RI presentes na pituitária e supra-renal, pode induzir diretamente a secreção de ACTH e glicocorticóide (MOHN et al., 2011). Ademais, tem sido observado que a IL-1 $\beta$ promove uma redução na neurogênese hipocampal, bem como na ativação de receptores alfa-amino-3-hidroxi-metil-5-4-isoxazolpropiónico (AMPA) que estão envolvidos na formação da memória e aprendizado, sendo que, no geral, as citocinas pró-inflamatórias levam a uma redução nos níveis de fator neurotrófico derivado do cérebro (BDNF) no hipocampo e córtex pré-frontal de animais submetidos a modelos de estresse crônico (WOHLEB et al., 2016).

O fator nuclear kappa B (NF-kB) pode se ligar a regiões promotoras do gene $\mathrm{CRH}$ levando a sua ativação, dessa forma o aumento de citocinas pró-inflamatórias como IL-1 $\beta$, IL-6 e TNF- $\alpha$ pode levar a ativação do eixo HPA uma vez que são ativadoras da via de sinalização do NF-kB (KAGEYAMA et al., 2010).

O estresse oxidativo apresenta um importante papel no desenvolvimento de doenças neurodegenerativas e da depressão, a exposição ao estresse crônico pode interferir nos sistemas antioxidantes, tanto os enzimáticos, como superóxido dismutase (SOD) e catalase, bem como os não enzimáticos como glutationa (GSH), no mais, foi observado um aumento de espécies reativas de oxigênio (ROS) em pacientes com depressão maior (BILICl et al., 2001; LUCCA et al., 2009).

O óxido nítrico (NO) é um radical livre com funções de sinalização no SNC e periférico, sendo produzido pela enzima óxido nítrico sintase (NOS), durante a conversão da L-arginina para L-citrulina. Existem três isoformas de NOS descritas, a 
NOS neuronal (NOSn) principal forma expressa no encéfalo (BREDT; SNYDER, 1994; MORENO-LÓPEZ et al., 2000), a NOS endotelial (NOSe) e a NOS induzida (NOSi) (PAAKKARI; LINDSBERG, 1995). No SNC, o NO desempenha funções fisiológicas e patológicas importantes, modulando a atividade sináptica durante a potenciação de longo prazo (LTP), a neurogênese, a sinaptogênese, processos de neurodegeneração, sobrevivência e diferenciação neuronal (CHO et al., 2009; ZHOU; ZHU, 2009).

Diferentes trabalhos tem relacionado o papel da NOSn com doenças psiquiátricas tais como, depressão (ZHOU; ZHU, 2009), ansiedade (ZHANG et al., 2010), esquizofrenia (O'DONOVAN et al., 2008; SHINKAl et al., 2002) e agressividade (CHIAVEGATTO et al., 2001). O estresse crônico é um fator de risco para o desenvolvimento de depressão em indivíduos com vulnerabilidade genética (TSANKOVA et al., 2006; WARNER-SCHMIDT; DUMAN, 2006), devido a hiperatividade do eixo HPA, que se correlaciona diretamente com a função prejudicada dos receptores GR no hipocampo, córtex e hipotálamo sendo um importante fator etiológico no desenvolvimento de doenças psiquiátricas (BOYLE et al., 2005). Além disso, vários estudos têm indicado que inibidores da NOS apresentam propriedades semelhantes a antidepressivos, sob condições fisiológicas (JOCA; GUIMARÃES, 2006).

Outro importante ponto é a relação que tem sido observada entre o aumento da atividade da NOSn e a redução de receptores de GR no hipocampo de camundongos (ZHOU et al., 2011). Níveis elevados de glicocorticóides induzem um aumento na expressão de NOSn no hipocampo via ativação de receptores MR, e por sua vez, promove uma redução nos níveis de GR na região hipocampal, com consequente ativação da via da ERK (ZHOU et al., 2011).

\subsection{Ouabaína}

A ouabaína (OUA) é um glicosídeo cardiotônico esteroidal classicamente conhecido por inibir a $\mathrm{Na}^{+}, \mathrm{K}^{+}$-ATPase (NKA). A OUA foi primeiramente descrita como um composto de origem vegetal e utilizada para tratamento da insuficiência cardíaca (BLAUSTEIN, 1993), sendo posteriormente descrita como um componente endógeno presente em mamíferos superiores (HAMLYN et al., 1991). Este digitálico pode ser 
encontrado no plasma humano em concentrações nanomolares, sendo produzido pela adrenal, hipotálamo e hipófise (FERRANDI et al., 1997; HAMLYN et al., 1991), e apresentando características químico-estruturais, biológicas e imunológicas comparáveis as da OUA encontrada em vegetais (FERRANDI et al., 1997; HAMLYN et al., 1991; SCHONER, 2000). Evidências sugerem que a síntese de OUA pode ser modificada de acordo com o estado fisiológico do organismo. Níveis elevados deste digitálico foram encontrados em pacientes hipertensos (SCHONER, 2000), assim como em diferentes modelos de ratos com hipertensão (HUANG; LEENEN, 1996). A OUA também participa da resposta do organismo ao estresse agudo, onde o exercício físico é capaz de aumentar seus níveis em ratos, cachorros e seres humanos minutos após o início da atividade física (GOTO et al., 1992).

Também tem sido demonstrado o efeito modulatório da OUA em eventos como, o crescimento celular, migração e morte celular programada (ABRAMOWITZ et al., 2003; BARWE et al., 2005; LIU et al., 2004) por meio da enzima NKA, que além de seu papel regulatório na homeostasia iônica, desempenha um papel na transdução de sinal e na ativação da transcrição gênica (KOMETIANI et al., 1998; PENG et al., 1996; TIAN; GONG; XIE, 2001; XIE; ASKARI, 2002).

Uma das vias ativadas pela interação entre a NKA e a OUA é a via IP3/Ca ${ }^{2+}$ (Inositol-trifosfato/Cálcio). Quando a OUA se liga a NKA promove uma mudança conformacional que favorece a interação direta de uma região conservada da porção $\mathrm{N}$ - terminal da subunidade $\alpha$ com a porção $\mathrm{N}$-terminal do receptor de canal de $\mathrm{Ca}^{2+}$ IP3R, presente no retículo endoplasmático, resultando em abertura e fechamento ritmados do canal. A OUA, também é capaz de promover a oscilação de $\mathrm{Ca}^{2+}$, por via dos canais de $\mathrm{Ca}^{2+}$-voltagem dependente (AIZMAN; APERIA, 2003; ZHANG et al., 2006).

Além da sinalização por $\mathrm{Ca}^{2+}$, a enzima NKA é capaz de ativar a via da Src quinase (CEREIJIDO et al., 2012; TIAN et al., 2006), via interação proteína-proteína, levando a consequente transativação do EGFR (receptor do fator de crescimento epidermal), e ativação da via da MAPK (proteína quinase ativada por mitógeno) (CEREIJIDO et al., 2012). A via da MAPK, por sua vez pode ativar vários fatores de transcrição, tais como CREB (elemento de resposta ligado ao AMP cíclico) e NF-кB, os quais estão associados transcrição de inúmeros fatores neurotróficos e citocinas (AIZMAN; APERIA, 2003). A NKA parece ainda modular a liberação de glutamato e a 
expressão de receptores N-Metil-D-Aspartato (NMDA) (BERSIER; RODRIGUEZ DE LORES ARNAIZ, 2009; KAWAMOTO et al., 2012).

Estudos recentes realizados em nosso laboratório mostraram que a administração intra-hipocampal de $10 \mathrm{nM}$ de OUA em ratos aumentou, após 1 hora, a expressão de genes associados à sinalização inflamatória e fator neurotrófico derivado de cérebro (BDNF) e possível neuroproteção pela modulação do fator nuclear kB (NF-kB). Essa modulação se deve em parte pela ação dos receptores glutamatérgicos tipo NMDA (N-metil-D-Aspartato) (KAWAMOTO et al., 2012). Além disso, estudos em cultura de células primárias de cerebelo de rato mostraram 0 envolvimento da via NMDA-Src-Ras-MAPK na ativação do NF-kB e na modulação destes genes pela OUA (DE SA LIMA et al., 2013). Os resultados desses estudos confirmam a existência de um novo tipo de sinalização para a OUA no SNC (KAWAMOTO et al., 2012). Ademais, foi demonstrado em modelo, in vivo, que a injeção intra-hipocampal de OUA $10 \mathrm{nM}$ promoveu um aumento na ramificação dendrítica, bem como melhorou a memória espacial e prejudicou a extinção da memória operacional e estes efeitos foram decorrentes da ativação das vias de sinalização, importantes na regulação de sinapses, CREB-BDNF, NF-KB, GSK-3ß e Wnt/B-Catenina (ORELLANA et al., 2018).

Interessantemente, estudos tem demonstrado a participação de compostos semelhantes aos digitálicos no desenvolvimento da depressão, bem como foi observado alterações genéticas nas isoformas alfas da NKA em pacientes com transtorno bipolar (GOLDSTEIN et al., 2009, 2012). Diante disto, nosso trabalho teve como objetivo avaliar o papel do tratamento crônico imprevisível com OUA em ratos submetidos ao estresse crônico imprevisível. 


\section{CONCLUSÃO}

Nossos resultados em conjunto podem concluir que o tratamento intermitente com OUA é eficiente em reverter a hiperatividade do eixo HPA induzido pelo CUS, por meio da redução de glicocorticoide, redução na expressão de CRH-CRHR1, aumento do CRHR2, bem como diminuir a neuroinflamação de baixo grau induzido pelo CUS, e aumentar os níveis de BDNF. Essas alterações bioquímicas contribuíram para uma reversão nos prejuízos na memória de longo prazo induzida pelo CUS. Além disso, concluímos que o CUS, bem como o tratamento apenas com OUA promovem uma melhora na memória emocional, que foi constatado no teste comportamental de condicionamento da memória ao medo, estes resultados sugerem que o protocolo do CUS de 14 dias promove uma adaptação neuronal levando a redesignação da memória ao medo, aqui configurado pelo choque, e o tratamento com a OUA abrevia esse processo. 
Figura 27 - Figura ilustrativa das conclusões do estudo.

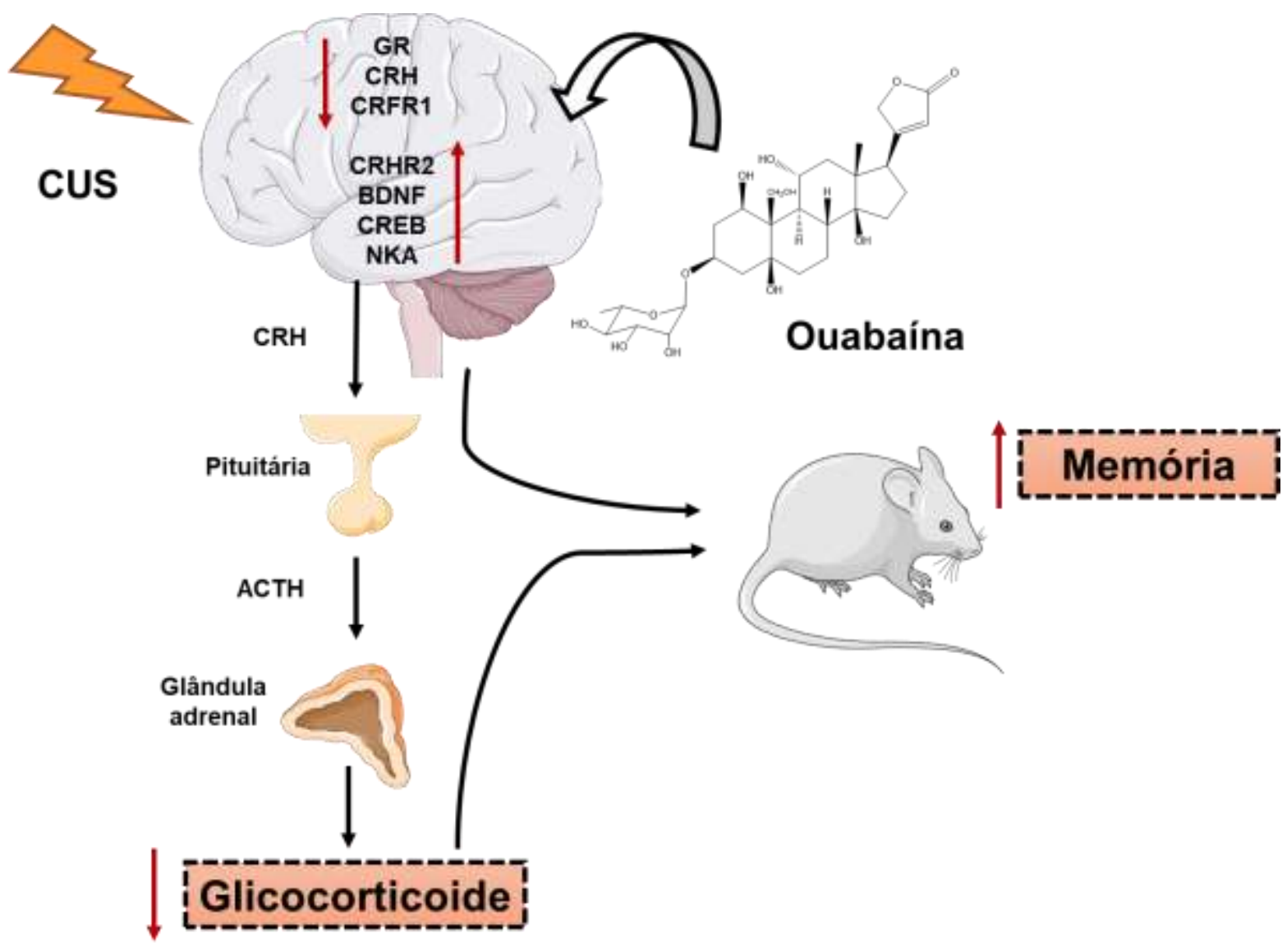

O protocolo de estresse crônico imprevisível (CUS) promoveu uma hiperatividade do eixo HPA, com redução na expressão de $\mathrm{GR}$, aumento na expressão de $\mathrm{CRH}$, aumento nos níveis de corticosterona, aumento na atividade da iNOS e da NKA, estes efeitos bioquímicos foram refletidos em prejuízos na memória no teste de reconhecimento do objeto novo. Porém o CUS promoveu uma melhora na memória emocional dos animais. O tratamento intermitente com Ouabaína reverte os efeitos do estresse crônico imprevisível (CUS) via redução na expressão de $\mathrm{CRH}-\mathrm{CRHR} 1$, aumento da atividade de NKA, redução na atividade da iNOS, aumento da via CREB-BDNF e dos níveis dos receptores $\mathrm{CRH} 2$, promovendo uma melhora na memória de longo prazo e emocional dos animais, possivelmente pela modulação das vias propostas assim como pela diminuição dos níveis de glicocorticoide. 


\section{REFERÊNCIAS}

ABRAMOWITZ, J. et al. Ouabain- and Marinobufagenin-Induced Proliferation of Human Umbilical Vein Smooth Muscle Cells and a Rat Vascular Smooth Muscle Cell Line, A7r5. Circulation, 2003.

AIZMAN, O.; APERIA, A. Na,K-ATPase as a signal transducer. Annals of the New York Academy of Sciences, v. 986, p. 489-496, abr. 2003.

ALLEN, S. J.; DAWBARN, D. Clinical relevance of the neurotrophins and their receptors. Clinical science (London, England: 1979), v. 110, n. 2, p. 175-191, fev. 2006.

ANACKER, C. et al. Glucocorticoid-related molecular signaling pathways regulating hippocampal neurogenesis. Neuropsychopharmacology: official publication of the American College of Neuropsychopharmacology, v. 38, n. 5, p. 872-883, abr. 2013.

ANDRADE, L. H. et al. Mental disorders in megacities: findings from the Sao Paulo megacity mental health survey, Brazil. PloS one, v. 7, n. 2, p. e31879, 2012.

ANTUNES, M.; BIALA, G. The novel object recognition memory: neurobiology, test procedure, and its modifications. Cognitive processing, v. 13 , n. 2, p. 93-110, maio 2012.

BAHR, B. A. et al. Mouse telencephalon exhibits an age-related decrease in glutamate (AMPA) receptors but no change in nerve terminal markers. Brain research, v. 589, $n$. 2, p. 320-326, set. 1992.

BALE, T. L. et al. Mice deficient for corticotropin-releasing hormone receptor-2 display anxiety-like behaviour and are hypersensitive to stress. Nature genetics, v. 24, n. 4, p. 410-414, abr. 2000.

BALE, T. L.; VALE, W. W. CRF AND CRF R ECEPTORS : Role in Stress Responsivity and Other Behaviors. Annual Review of Pharmacology and Toxicology, 2004.

BARKER, G. R. I. et al. Recognition memory for objects, place, and temporal order: a disconnection analysis of the role of the medial prefrontal cortex and perirhinal cortex. The Journal of neuroscience : the official journal of the Society for Neuroscience, v. 27, n. 11, p. 2948-2957, mar. 2007.

BARNUM, C. J. et al. Psychological stress in adolescent and adult mice increases neuroinflammation and attenuates the response to LPS challenge. Journal of neuroinflammation, v. 9, p. 9, jan. 2012.

BARWE, S. P. et al. Novel role for Na,K-ATPase in phosphatidylinositol 3-kinase signaling and suppression of cell motility. Molecular biology of the cell, v. 16, n. 3, p. 1082-1094, mar. 2005.

BELDA, X. et al. Exposure to severe stressors causes long-lasting dysregulation of 
resting and stress-induced activation of the hypothalamic-pituitary-adrenal axis. Annals of the New York Academy of Sciences. Anais...2008

BERSIER, M. G.; RODRIGUEZ DE LORES ARNAIZ, G. Intracerebroventricular administration of ouabain to rats changes the expression of NMDA receptor subunits in cerebral cortex and hippocampus. Neurochemical research, v. 34, n. 9, p. 16501657, set. 2009.

BERTON, O.; NESTLER, E. J. New approaches to antidepressant drug discovery: Beyond monoamines. Nature Reviews Neuroscience, 2006.

BESEDOVSKY, H. O.; DEL REY, A. Immune-neuro-endocrine interactions: Facts and hypotheses Endocrine Reviews, 1996.

BILICI, M. et al. Antioxidative enzyme activities and lipid peroxidation in major depression: Alterations by antidepressant treatments. Journal of Affective Disorders, 2001.

BITTENCOURT, J. C. et al. Urocortin expression in rat brain: evidence against a pervasive relationship of urocortin-containing projections with targets bearing type 2 CRF receptors. The Journal of comparative neurology, v. 415, n. 3, p. 285-312, dez. 1999.

BLANK, T. et al. Corticotropin-releasing factor receptors couple to multiple G-proteins to activate diverse intracellular signaling pathways in mouse hippocampus: role in neuronal excitability and associative learning. The Journal of neuroscience : the official journal of the Society for Neuroscience, 2003.

BLAUSTEIN, M. P. Physiological effects of endogenous ouabain: control of intracellular $\mathrm{Ca}^{2+}$ stores and cell responsiveness. The American journal of physiology, 1993.

BOORSE, G. C.; DENVER, R. J. Widespread tissue distribution and diverse functions of corticotropin-releasing factor and related peptides. General and comparative endocrinology, v. 146, n. 1, p. 9-18, mar. 2006.

BOYLE, M. P. et al. Acquired deficit of forebrain glucocorticoid receptor produces depression-like changes in adrenal axis regulation and behavior. Proceedings of the National Academy of Sciences, 2005.

BREDT, D. S.; SNYDER, S. H. Transient nitric oxide synthase neurons in embryonic cerebral cortical plate, sensory ganglia, and olfactory epithelium. Neuron, 1994.

CEREIJIDO, M. et al. The $\mathrm{Na}^{+}-\mathrm{K}^{+}-\mathrm{ATP}$ ase as self-adhesion molecule and hormone receptor. American journal of physiology. Cell physiology, v. 302, n. 3, p. C473-81, fev. 2012.

CHARMANDARI, E.; TSIGOS, C.; CHROUSOS, G. ENDOCRINOLOGY OF THE STRESS RESPONSE 1. Annu. Rev. Physiol, 2005.

CHESNOKOVA, V.; MELMED, S. Minireview: Neuro-immuno-endocrine modulation of the hypothalamic-pituitary-adrenal (HPA) axis by gp130 signaling molecules. Endocrinology, v. 143, n. 5, p. 1571-1574, maio 2002. 
CHIAVEGATTO, S. et al. Brain serotonin dysfunction accounts for aggression in male mice lacking neuronal nitric oxide synthase. Proceedings of the National Academy of Sciences of the United States of America, 2001.

$\mathrm{CHO}$, D.-H. H. et al. S-nitrosylation of Drp1 mediates beta-amyloid-related mitochondrial fission and neuronal injury. Science, 2009.

CLARKE, J. R. et al. Plastic modifications induced by object recognition memory processing. Proceedings of the National Academy of Sciences of the United States of America, v. 107, n. 6, p. 2652-2657, fev. 2010.

COHEN, S.; JANICKI-DEVERTS, D.; MILLER, G. E. Psychological stress and disease. JAMA, v. 298, n. 14, p. 1685-1687, out. 2007.

COPPEN, A. et al. Mineral metabolism in mania. British medical journal, v. 1, n. 5479, p. 71-75, jan. 1966.

CUNHA, G. M. A. et al. Increased density and synapto-protective effect of adenosine A2A receptors upon sub-chronic restraint stress. Neuroscience, v. 141, n. 4, p. 17751781, set. 2006.

DE KLOET, E. R. et al. Brain corticosteroid receptor balance in health and disease. Endocrine Reviews, 1998.

DE KLOET, E. R. et al. Glucocorticoid signaling and stress-related limbic susceptibility pathway: About receptors, transcription machinery and microRNA. Brain Research, 2009.

DE SA LIMA, L. et al. Ouabain activates NFkappaB through an NMDA signaling pathway in cultured cerebellar cells. Neuropharmacology, v. 73, p. 327-336, out. 2013.

DELGADO, M. R.; OLSSON, A.; PHELPS, E. A. Extending animal models of fear conditioning to humans. Biological psychology, v. 73, n. 1, p. 39-48, jul. 2006.

DINKEL, K.; MACPHERSON, A.; SAPOLSKY, R. M. Novel glucocorticoid effects on acute inflammation in the CNS. Journal of Neurochemistry, 2003.

DUCLOT, F.; KABBAJ, M. The Role of Early Growth Response 1 (EGR1) in Brain Plasticity and Neuropsychiatric Disorders. Frontiers in Behavioral Neuroscience, 2017.

EL-MALLAKH, R. S.; WYATT, R. J. The Na,K-ATPase hypothesis for bipolar illness. Biological psychiatry, v. 37, n. 4, p. 235-244, fev. 1995.

ESMANN, M. ATPase and phosphatase activity of $\mathrm{Na}^{+}, \mathrm{K}^{+}-\mathrm{ATPase}$ : molar and specific activity, protein determination. Methods in enzymology, v. 156, p. 105-115, 1988.

FERRANDI, M. et al. Ouabain-like factor quantification in mammalian tissues and plasma: Comparison of two independent assays. Hypertension, 1997.

FESCHENKO, M. S.; SWEADNER, K. J. Conformation-dependent phosphorylation of $\mathrm{Na}, \mathrm{K}-\mathrm{ATPase}$ by protein kinase $\mathrm{A}$ and protein kinase $\mathrm{C}$. The Journal of biological chemistry, v. 269, n. 48, p. 30436-30444, dez. 1994.

FOCKING, M.; HOLKER, I.; TRAPP, T. Chronic glucocorticoid receptor activation impairs CREB transcriptional activity in clonal neurons. Biochemical and biophysical 
research communications, v. 304, n. 4, p. 720-723, maio 2003.

FUCHS, E.; FLÜGGE, G. Chronic social stress: Effects on limbic brain structures. Physiology and Behavior. Anais...2003

FUERSTENWERTH, H. On the differences between ouabain and digitalis glycosides. American journal of therapeutics, v. 21, n. 1, p. 35-42, 2014.

GADEK-MICHALSKA, A. et al. Cytokines, prostaglandins and nitric oxide in the regulation of stress-response systems. Pharmacological reports: PR, v. 65, n. 6, p. 1655-1662, 2013.

GAFFORD, G. M. et al. Cell-type specific deletion of GABA(A)alpha1 in corticotropinreleasing factor-containing neurons enhances anxiety and disrupts fear extinction. Proceedings of the National Academy of Sciences of the United States of America, v. 109, n. 40, p. 16330-16335, out. 2012.

GAMARO, G. D. et al. Reduction of hippocampal $\mathrm{Na}+, \mathrm{K}+-\mathrm{ATPase}$ activity in rats subjected to an experimental model of depression. Neurochemical research, v. 28, n. 9, p. 1339-1344, set. 2003.

GARCIA, I. J. P. et al. Ouabain attenuates oxidative stress and modulates lipid composition in hippocampus of rats in lipopolysaccharide-induced hypocampal neuroinflammation in rats. Journal of cellular biochemistry, set. 2018.

GILLESPIE, C. F.; NEMEROFF, C. B. Hypercortisolemia and depression. Psychosomatic Medicine, 2005.

GOLDSTEIN, I. et al. Involvement of $\mathrm{Na}(+), \mathrm{K}(+)$-ATPase and endogenous digitalislike compounds in depressive disorders. Biological psychiatry, v. 60, n. 5, p. 491-499, set. 2006.

GOLDSTEIN, I. et al. Association Between Sodium- and Potassium-Activated Adenosine Triphosphatase a Isoforms and Bipolar Disorders. Biological Psychiatry, 2009.

GOLDSTEIN, I. et al. Neutralization of endogenous digitalis-like compounds alters catecholamines metabolism in the brain and elicits anti-depressive behavior. European neuropsychopharmacology: the journal of the European College of Neuropsychopharmacology, v. 22, n. 1, p. 72-79, jan. 2012.

GOLDWATER, D. S. et al. Structural and functional alterations to rat medial prefrontal cortex following chronic restraint stress and recovery. Neuroscience, v. 164, n. 2, p. 798-808, dez. 2009.

GOSHEN, I. et al. Brain interleukin-1 mediates chronic stress-induced depression in mice via adrenocortical activation and hippocampal neurogenesis suppression. Molecular psychiatry, v. 13, n. 7, p. 717-728, jul. 2008.

GOSHEN, I.; YIRMIYA, R. Interleukin-1 (IL-1): a central regulator of stress responses. Frontiers in neuroendocrinology, v. 30, n. 1, p. 30-45, jan. 2009.

GOTO, A. et al. Physiology and pharmacology of endogenous digitalis-like factors. Pharmacological reviews, v. 44, n. 3, p. 377-399, set. 1992. 
GOTO, A. et al. Stress-induced elevation of ouabainlike compound in rat plasma and adrenal. Hypertension (Dallas, Tex. : 1979), v. 26, n. 6 Pt 2, p. 1173-1176, dez. 1995.

GOULART, B. K. et al. Ketamine impairs recognition memory consolidation and prevents learning-induced increase in hippocampal brain-derived neurotrophic factor levels. Neuroscience, v. 167, n. 4, p. 969-973, jun. 2010.

GRANT, B. F. et al. Prevalence, correlates, co-morbidity, and comparative disability of DSM-IV generalized anxiety disorder in the USA: results from the National Epidemiologic Survey on Alcohol and Related Conditions. Psychological medicine, v. 35, n. 12, p. 1747-1759, dez. 2005.

GRIPPO, A. J. et al. Neuroendocrine and cytokine profile of chronic mild stressinduced anhedonia. Physiology \& behavior, v. 84, n. 5, p. 697-706, abr. 2005.

GRONLI, J. et al. Chronic mild stress inhibits BDNF protein expression and CREB activation in the dentate gyrus but not in the hippocampus proper. Pharmacology, biochemistry, and behavior, v. 85, n. 4, p. 842-849, dez. 2006.

HAMLYN, J. M. et al. Identification and characterization of a ouabain-like compound from human plasma. Biochemistry, 1991.

HANSEN, M. B.; NIELSEN, S. E.; BERG, K. Re-examination and further development of a precise and rapid dye method for measuring cell growth/cell kill. Journal of immunological methods, v. 119, n. 2, p. 203-210, maio 1989.

HELDT, S. A. et al. Hippocampus-specific deletion of BDNF in adult mice impairs spatial memory and extinction of aversive memories. Molecular psychiatry, v. 12, n. 7, p. 656-670, jul. 2007.

HENCKENS, M. J. A. G.; DEUSSING, J. M.; CHEN, A. Region-specific roles of the corticotropin-releasing factor-urocortin system in stress. Nature reviews. Neuroscience, v. 17, n. 10, p. 636-651, out. 2016.

HERMAN, J. P.; ADAMS, D.; PREWITT, C. Regulatory changes in neuroendocrine stress-integrative circuitry produced by a variable stress paradigm. Neuroendocrinology, v. 61, n. 2, p. 180-190, fev. 1995.

HUANG, B. S.; LEENEN, F. H. Brain "ouabain" and angiotensin II in salt-sensitive hypertension in spontaneously hypertensive rats. Hypertension (Dallas, Tex. : 1979), v. 28, n. 6, p. 1005-1012, dez. 1996.

HUNG, H. C. et al. CRF increases protein phosphorylation and enhances retention performance in rats. Neuroreport, v. 3, n. 2, p. 181-184, fev. 1992.

ITOI, K. et al. Microinjection of norepinephrine into the paraventricular nucleus of the hypothalamus stimulates corticotropin-releasing factor gene expression in conscious rats. Endocrinology, 1994.

JOCA, S. R. L.; GUIMARÃES, F. S. Inhibition of neuronal nitric oxide synthase in the rat hippocampus induces antidepressant-like effects. Psychopharmacology, 2006.

KAGEYAMA, K. et al. Cytokines induce NF-KB, Nurr1 and corticotropin-releasing factor gene transcription in Hypothalamic 4B cells. NeurolmmunoModulation, 2010. 
KALLARACKAL, A. J. et al. Chronic stress induces a selective decrease in AMPA receptor-mediated synaptic excitation at hippocampal temporoammonic-CA1 synapses. The Journal of neuroscience: the official journal of the Society for Neuroscience, v. 33, n. 40, p. 15669-15674, out. 2013.

KANDEL, E. R. The molecular biology of memory storage: a dialogue between genes and synapses. Science (New York, N.Y.), v. 294, n. 5544, p. 1030-1038, nov. 2001.

KAWAMOTO, E. M. et al. Influence of $\mathrm{N}$-methyl-D-aspartate receptors on ouabain activation of nuclear factor-kappaB in the rat hippocampus. Journal of neuroscience research, v. 90, n. 1, p. 213-228, jan. 2012.

KINOSHITA, P. F. et al. Signaling function of Na,K-ATPase induced by ouabain against LPS as an inflammation model in hippocampus. Journal of neuroinflammation, v. 11, p. 218, dez. 2014.

KOMETIANI, P. et al. Multiple signal transduction pathways link $\mathrm{Na}+\mathrm{K}+-\mathrm{ATPase}$ to growth-related genes in cardiac myocytes. The roles of Ras and mitogen-activated protein kinases. The Journal of biological chemistry, v. 273, n. 24, p. 15249-15256, jun. 1998.

KUMAR, B.; KUHAD, A.; CHOPRA, K. Neuropsychopharmacological effect of sesamol in unpredictable chronic mild stress model of depression: Behavioral and biochemical evidences. Psychopharmacology, 2011.

LANTE, F. et al. Low-frequency stimulation induces a new form of LTP, metabotropic glutamate (mGlu5) receptor- and PKA-dependent, in the CA1 area of the rat hippocampus. Hippocampus, v. 16, n. 4, p. 345-360, 2006.

LAREDO, J.; HAMILTON, B. P.; HAMLYN, J. M. Ouabain is secreted by bovine adrenocortical cells. Endocrinology, v. 135, n. 2, p. 794-797, ago. 1994.

LEITE, J. A. et al. Ouabain Modulates Zymosan-Induced Peritonitis in Mice. Mediators of inflammation, v. 2015, p. 265798, 2015.

LIGHTMAN, S. L. et al. The significance of glucocorticoid pulsatilityEuropean Journal of Pharmacology, 2008.

LISSEK, S. et al. Classical fear conditioning in the anxiety disorders: a meta-analysis. Behaviour research and therapy, v. 43, n. 11, p. 1391-1424, nov. 2005.

LIU, L. et al. Role of caveolae in ouabain-induced proliferation of cultured vascular smooth muscle cells of the synthetic phenotype. American journal of physiology. Heart and circulatory physiology, v. 287, n. 5, p. H2173-82, nov. 2004.

LIU, X.-L. et al. Fluoxetine regulates mTOR signalling in a region-dependent manner in depression-like mice. Scientific reports, v. 5, p. 16024, nov. 2015.

LOONEY, S. W.; EL-MALLAKH, R. S. Meta-analysis of erythrocyte Na,K-ATPase activity in bipolar illness. Depression and anxiety, v. 5, n. 2, p. 53-65, 1997.

LUCCA, G. et al. Effects of chronic mild stress on the oxidative parameters in the rat brain. Neurochemistry International, 2009. 
MACPHERSON, A.; DINKEL, K.; SAPOLSKY, R. Glucocorticoids worsen excitotoxininduced expression of pro-inflammatory cytokines in hippocampal cultures. Experimental Neurology, 2005.

MAES, M. et al. A review on the oxidative and nitrosative stress (O\&NS) pathways in major depression and their possible contribution to the (neuro)degenerative processes in that illnessProgress in Neuro-Psychopharmacology and Biological Psychiatry, 2011.

MAJZOUB, J. A. Corticotropin-releasing hormone physiology. European Journal of Endocrinology, 2006.

MARQUES, A. H.; SILVERMAN, M. N.; STERNBERG, E. M. Glucocorticoid dysregulations and their clinical correlates: From receptors to therapeutics. Annals of the New York Academy of Sciences. Anais...2009

MARTINS, J. M.; BANKS, W. A.; KASTIN, A. J. Acute modulation of active carriermediated brain-to-blood transport of corticotropin-releasing hormone. The American journal of physiology, v. 272, n. 2 Pt 1, p. E312-9, fev. 1997.

MATSUMOTO, $K$. et al. Social isolation stress down-regulates cortical early growth response 1 (Egr-1) expression in mice. Neuroscience research, v. 73, n. 3, p. 257262, jul. 2012.

MCKEE, M.; SCAVONE, C.; NATHANSON, J. A. Nitric oxide, cGMP, and hormone regulation of active sodium transport. Proceedings of the National Academy of Sciences of the United States of America, v. 91, n. 25, p. 12056-12060, dez. 1994.

MILLER, G. E. et al. A functional genomic fingerprint of chronic stress in humans: blunted glucocorticoid and increased NF-kappaB signaling. Biological psychiatry, $\mathrm{v}$. 64, n. 4, p. 266-272, ago. 2008.

MOHN, C. E. et al. Adrenal gland responses to lipopolysaccharide after stress and ethanol administration in male rats. Stress, 2011.

MONTEGGIA, L. M. et al. Essential role of brain-derived neurotrophic factor in adult hippocampal function. Proceedings of the National Academy of Sciences of the United States of America, v. 101, n. 29, p. 10827-10832, jul. 2004.

MORENO-LÓPEZ, B. et al. Morphological bases for a role of nitric oxide in adult neurogenesis1. Brain Research, 2000.

MUNHOZ, C. D. Chronic Unpredictable Stress Exacerbates LipopolysaccharideInduced Activation of Nuclear Factor- B in the Frontal Cortex and Hippocampus via Glucocorticoid Secretion. Journal of Neuroscience, 2006.

MUNHOZ, C. D. et al. Glucocorticoids Exacerbate Lipopolysaccharide-Induced Signaling in the Frontal Cortex and Hippocampus in a Dose-Dependent Manner. Journal of Neuroscience, 2010.

NADEAU, S.; RIVEST, S. Glucocorticoids play a fundamental role in protecting the brain during innate immune response. The Journal of neuroscience : the official journal of the Society for Neuroscience, 2003.

NG, W. X. D. et al. Neurobiological evidence for thalamic, hippocampal and related 
glutamatergic abnormalities in bipolar disorder: a review and synthesis. Neuroscience and biobehavioral reviews, v. 33, n. 3, p. 336-354, mar. 2009.

NICOLETTI, V. G. et al. AMPA-selective glutamate receptor subunits in the rat hippocampus during aging. Journal of neuroscience research, v. 40, n. 2, p. 220-224, fev. 1995.

NUMAKAWA, T. et al. Glucocorticoid receptor interaction with TrkB promotes BDNFtriggered PLC-gamma signaling for glutamate release via a glutamate transporter. Proceedings of the National Academy of Sciences of the United States of America, $v$. 106, n. 2, p. 647-652, jan. 2009.

NURNBERGER, J. J. et al. Red cell ouabain-sensitive Na+-K+-adenosine triphosphatase: a state marker in affective disorder inversely related to plasma cortisol. Biological psychiatry, v. 17, n. 9, p. 981-992, set. 1982.

O'DONOVAN, M. C. et al. Identification of loci associated with schizophrenia by genome-wide association and follow-up. Nature Genetics, 2008.

OLIVEIRA, A. M. M. et al. Post-training reversible inactivation of the hippocampus enhances novel object recognition memory. Learning \& memory (Cold Spring Harbor, N.Y.), v. 17, n. 3, p. 155-160, mar. 2010.

ORELLANA, A. M. et al. Ouabain increases neuronal branching in hippocampus and improves spatial memory. Neuropharmacology, v. 140, p. 260-274, set. 2018.

OSSOWSKA, K. et al. Decline in motor functions in aging is related to the loss of NMDA receptors. Brain research, v. 907, n. 1-2, p. 71-83, jul. 2001.

PAAKKARI, I.; LINDSBERG, P. Nitric oxide in the central nervous system. Annals of medicine, v. 27, n. 3, p. 369-377, jun. 1995.

PALUMBO, M. L. et al. Loss of hippocampal neuronal nitric oxide synthase contributes to the stress-related deficit in learning and memory. Journal of neurochemistry, v. 102, n. 1, p. 261-274, jul. 2007.

PANG, P. T.; LU, B. Regulation of late-phase LTP and long-term memory in normal and aging hippocampus: role of secreted proteins tPA and BDNF. Ageing research reviews, v. 3, n. 4, p. 407-430, nov. 2004.

PAPADIMITRIOU, A.; PRIFTIS, K. N. Regulation of the hypothalamic-pituitary-adrenal axisNeurolmmunoModulation, 2009.

PEEN, J. et al. The current status of urban-rural differences in psychiatric disorders. Acta psychiatrica Scandinavica, v. 121, n. 2, p. 84-93, fev. 2010.

PENG, M. et al. Partial inhibition of $\mathrm{Na+/K+-ATPase} \mathrm{by} \mathrm{ouabain} \mathrm{induces} \mathrm{the} \mathrm{Ca2+-}$ dependent expressions of early-response genes in cardiac myocytes. The Journal of biological chemistry, v. 271, n. 17, p. 10372-10378, abr. 1996.

RADLEY, J. J. et al. Repeated stress alters dendritic spine morphology in the rat medial prefrontal cortex. The Journal of comparative neurology, v. 507, n. 1, p. 1141-1150, mar. 2008. 
RADULOVIC, J. et al. Modulation of learning and anxiety by corticotropin-releasing factor (CRF) and stress: differential roles of CRF receptors 1 and 2. The Journal of neuroscience : the official journal of the Society for Neuroscience, v. 19, n. 12, p. 50165025, jun. 1999.

RAMOT, A. et al. Hypothalamic CRFR1 is essential for HPA axis regulation following chronic stress. Nature neuroscience, v. 20, n. 3, p. 385-388, mar. 2017.

REGER, M. L.; HOVDA, D. A.; GIZA, C. C. Ontogeny of Rat Recognition Memory measured by the novel object recognition task. Developmental psychobiology, v. 51, n. 8, p. 672-678, dez. 2009.

ROOZENDAAL, B. et al. Glucocorticoid enhancement of memory requires arousalinduced noradrenergic activation in the basolateral amygdala. Proceedings of the National Academy of Sciences of the United States of America, v. 103, n. 17, p. 67416746, abr. 2006.

SCHESCHOWITSCH, K.; LEITE, J. A.; ASSREUY, J. New insights in glucocorticoid receptor signaling-more than just a ligand-binding receptorFrontiers in Endocrinology, 2017.

SCHIAVONE, S. et al. Severe Life Stress and Oxidative Stress in the Brain: From Animal Models to Human Pathology. Antioxidants \& Redox Signaling, 2013.

SCHMIDT, H. D.; DUMAN, R. S. The role of neurotrophic factors in adult hippocampal neurogenesis, antidepressant treatments and animal models of depressive-like behavior. Behavioural pharmacology, v. 18, n. 5-6, p. 391-418, set. 2007.

SCHMIDT, M. V et al. Individual stress vulnerability is predicted by short-term memory and AMPA receptor subunit ratio in the hippocampus. The Journal of neuroscience: the official journal of the Society for Neuroscience, v. 30, n. 50, p. 16949-16958, dez. 2010.

SCHNELL, E. et al. Direct interactions between PSD-95 and stargazin control synaptic AMPA receptor number. Proceedings of the National Academy of Sciences of the United States of America, v. 99, n. 21, p. 13902-13907, out. 2002.

SCHONER, W. Ouabain, a new steroid hormone of adrenal gland and hypothalamus. Experimental and clinical endocrinology \& diabetes : official journal, German Society of Endocrinology [and] German Diabetes Association, v. 108, n. 7, p. 449-454, 2000.

SEVGI, S.; OZEK, M.; EROGLU, L. L-NAME prevents anxiety-like and depression-like behavior in rats exposed to restraint stress. Methods and findings in experimental and clinical pharmacology, v. 28, n. 2, p. 95-99, mar. 2006.

SHAW, D. M. Mineral metabolism, mania, and melancholia. British medical journal, v. 2, n. 5508, p. 262-267, jul. 1966.

SHINKAI, T. et al. Allelic association of the neuronal nitric oxide synthase (NOS1) gene with schizophrenia. Molecular Psychiatry, 2002.

SILVERMAN, M. N.; STERNBERG, E. M. Glucocorticoid regulation of inflammation and its functional correlates: From HPA axis to glucocorticoid receptor dysfunction Annals of the New York Academy of Sciences, 2012. 
SILVERS, J. M. et al. Automation of the novel object recognition task for use in adolescent rats. Journal of neuroscience methods, v. 166, n. 1, p. 99-103, out. 2007.

SUGAMA, S. et al. Microglial activation is inhibited by corticosterone in dopaminergic neurodegeneration. Journal of Neuroimmunology, 2009.

SUGAMA, S. et al. Corticosteroids limit microglial activation occurring during acute stress. Neuroscience, 2013.

TAMASHIRO, K. L. K.; NGUYEN, M. M. N.; SAKAI, R. R. Social stress: from rodents to primates. Frontiers in neuroendocrinology, v. 26, n. 1, p. 27-40, abr. 2005.

TAO, X. et al. Ca2+ influx regulates BDNF transcription by a CREB family transcription factor-dependent mechanism. Neuron, v. 20, n. 4, p. 709-726, abr. 1998.

TER HEEGDE, F.; DE RIJK, R. H.; VINKERS, C. H. The brain mineralocorticoid receptor and stress resiliencePsychoneuroendocrinology, 2015.

TIAN, J. et al. Binding of Src to $\mathrm{Na}+/ \mathrm{K}+-\mathrm{ATP}$ ase forms a functional signaling complex. Molecular biology of the cell, v. 17, n. 1, p. 317-326, jan. 2006.

TIAN, J.; GONG, X.; XIE, Z. Signal-transducing function of Na+-K+-ATPase is essential for ouabain's effect on [Ca2+]i in rat cardiac myocytes. American journal of physiology. Heart and circulatory physiology, v. 281, n. 5, p. H1899-907, nov. 2001.

TSANKOVA, N. M. et al. Sustained hippocampal chromatin regulation in a mouse model of depression and antidepressant action. Nature Neuroscience, 2006.

TSIGOS, C.; CHROUSOS, G. P. Hypothalamic-pituitary-adrenal axis, neuroendocrine factors and stress. Journal of Psychosomatic Research. Anais...2002

TURNBULL, A. V.; RIVIER, C. L. Regulation of the Hypothalamic-Pituitary-Adrenal Axis by Cytokines: Actions and Mechanisms of Action. Physiological Reviews, 1999.

ULRICH-LAI, Y. M. et al. Chronic stress induces adrenal hyperplasia and hypertrophy in a subregion-specific manner. American journal of physiology. Endocrinology and metabolism, v. 291, n. 5, p. E965-73, nov. 2006.

VALE, W. et al. Characterization of a 41-residue ovine hypothalamic peptide that stimulates secretion of corticotropin and $\beta$-endorphin. Science, 1981.

VASCONCELLOS, A. P. S. et al. Effect of chronic stress on spatial memory in rats is attenuated by lithium treatment. Physiology \& behavior, v. 79, n. 2, p. 143-149, jul. 2003.

WANG, D.; AN, S. C.; ZHANG, X. Prevention of chronic stress-induced depressionlike behavior by inducible nitric oxide inhibitor. Neuroscience letters, v. 433, n. 1, p. 59-64, mar. 2008.

WARNER-SCHMIDT, J. L.; DUMAN, R. S. Hippocampal neurogenesis: Opposing effects of stress and antidepressant treatment Hippocampus, 2006.

WATANABE, Y.; GOULD, E.; MCEWEN, B. S. Stress induces atrophy of apical dendrites of hippocampal CA3 pyramidal neurons. Brain Research, 1992. 
WOHLEB, E. S. et al. Integrating neuroimmune systems in the neurobiology of depression. Nature reviews. Neuroscience, v. 17, n. 8, p. 497-511, ago. 2016.

WONG, M. L. et al. Localization of urocortin messenger RNA in rat brain and pituitary. Molecular psychiatry, v. 1, n. 4, p. 307-312, set. 1996.

$\mathrm{XIE}, \mathrm{Z}$.; ASKARI, A. $\mathrm{Na}(+) / \mathrm{K}(+)$-ATPase as a signal transducer. European journal of biochemistry, v. 269, n. 10, p. 2434-2439, maio 2002.

YOU, Z. et al. Pro- and anti-inflammatory cytokines expression in rat's brain and spleen exposed to chronic mild stress: involvement in depression. Behavioural brain research, v. 225, n. 1, p. 135-141, nov. 2011.

YOUNG, E. A.; ABELSON, J.; LIGHTMAN, S. L. Cortisol pulsatility and its role in stress regulation and health. Front Neuroendocrinol, 2004.

YUEN, E. Y. et al. Repeated stress causes cognitive impairment by suppressing glutamate receptor expression and function in prefrontal cortex. Neuron, v. 73, n. 5, p. 962-977, mar. 2012.

ZHANG, J. et al. Neuronal Nitric Oxide Synthase Alteration Accounts for the Role of 5HT1A Receptor in Modulating Anxiety-Related Behaviors. Journal of Neuroscience, 2010.

ZHANG, S. et al. Distinct role of the N-terminal tail of the Na,K-ATPase catalytic subunit as a signal transducer. The Journal of biological chemistry, v. 281, n. 31, p. 2195421962, ago. 2006.

ZHOU, L.; ZHU, D.-Y. Neuronal nitric oxide synthase: Structure, subcellular localization, regulation, and clinical implications. Nitric Oxide, 2009.

ZHOU, Q.-G. et al. Hippocampal Neuronal Nitric Oxide Synthase Mediates the StressRelated Depressive Behaviors of Glucocorticoids by Downregulating Glucocorticoid Receptor. Journal of Neuroscience, 2011.

ZIEGLER, D. R. Neurocircuitry of Stress Integration: Anatomical Pathways Regulating the Hypothalamo-Pituitary-Adrenocortical Axis of the Rat. Integrative and Comparative Biology, 2002. 